\title{
QCD Sum Rules on the Lattice
}

\author{
Chris R. Allton ${ }^{\mathrm{a}}$ and Stefano Capitani ${ }^{\mathrm{b}}$ \\ a Department of Physics, University of Wales Swansea, U.K. \\ b DESY - Theory Group, Hamburg, Germany
}

We study the work of Leinweber by applying the Continuum Model of QCD Sum Rules (QCDSR) to the analysis of (quenched) lattice correlation functions. We expand upon his work in several areas and find that, while the QCDSR Continuum Model very adequately fits lattice data, it does so only for non-physical values of its parameters. The non-relativistic model is found to predict essentially the same form for the correlation functions as the QCDSR Continuum Model but without the latter's restrictions. By fitting lattice data to a general form which includes the non-relativistic quark model as a special case, we confirm it as the model of choice.

\section{INTRODUCTION}

Lattice Gauge Theory and QCD Sum Rules (QCDSR) are two areas of research which have been widely employed to deepen the understanding of systems governed by the strong interactions. In this paper we combine both methods following the pioneering work of Leinweber [1,2]. Utilising known results in QCD Sum Rules we apply them to Lattice Gauge Theory in order to check that the two are compatible.

We extend the work of [1,2] by including mesonic states, by applying our analysis to several $\beta$ values, and by studying data from both the Wilson and SW-Clover actions. We also study the non-relativistic quark model predictions.

The lattice data used is from the APE collaboration and is a mixture of (quenched) Wilson and SW-Clover with beta values ranging from $\beta=6.0$ to $\beta=6.4$. Zero-momentum 2-point hadronic correlation functions were studied for the nucleon, delta and the following mesonic operators: $P_{5}, A_{0}$ $\& V_{i}$ (in an obvious notation). A longer version of this work appears in [3].

\section{QCDSR CONTINUUM MODEL}

The quark propagator in Euclidean space's Wilson OPE reads (in the coordinate gauge),

$S_{q}^{a a^{\prime}}=\left\{\frac{\gamma \cdot x}{2 \pi^{2} x^{4}}+\frac{m_{q}}{(2 \pi x)^{2}}-\frac{\langle: \bar{q} q:\rangle}{2^{2} 3}\right\} \delta^{a a^{\prime}}+\cdots$

\footnotetext{
${ }^{*}$ Talk presented by C.R. Allton, Preprint SWAT/163
}

This is substituted into the (Wick contracted) 2point function leading to the generic form:

$$
G_{2}^{O P E}(t)=\sum_{n=-\infty}^{n_{0}} \frac{1}{t^{n}} \cdot C_{n} O_{n}\left(m_{q},\langle: \bar{q} q:\rangle\right)
$$

where $C_{n}$ is a numerical coefficient, $O_{n}$ is some operator function, and $n_{0}$ is a positive integer. It is now useful to express the timesliced correlation function, $G_{2}(t)$, in the spectral representation: $G_{2}(t)=\int_{0}^{\infty} \rho(s) e^{-s t} d s$. The OPE spectral density, $\rho O P E(s)$, is calculated by means of a simple inverse Laplace transform of $G_{2}^{O P E}$,

$\rho^{O P E}(s)=\sum_{n=1}^{n_{0}} \frac{s^{n-1}}{(n-1) !} \cdot C_{n} O_{n}\left(m_{q},\langle: \bar{q} q:\rangle\right)$,

where $\operatorname{Re}(t)>0$. In the sum over $n$, we have kept only the positive values of $n$ since we are interested in the leading terms as $t \rightarrow 0$.

The QCD Continuum Model is introduced by setting a threshold $s_{0}$ in the energy scale $s$, so that the excited states' contribution to $G_{2}(t)$ is given only by the energies above that scale. Performing the integral over $s$ we obtain,

$$
\begin{aligned}
G_{2}^{c o n t}(t) & \equiv \int_{s_{0}}^{\infty} \rho^{O P E}(s) e^{-s t} d s \\
& =e^{-s_{0} t} \sum_{n=1}^{n_{0}} \sum_{k=0}^{n-1} \frac{1}{k !} \frac{s_{0}^{k}}{t^{n-k}} \cdot C_{n} O_{n}
\end{aligned}
$$

The full correlation function contains the ground state as well as the above continuum contribution 
$G_{2}^{\text {cont }}$. This is simply included as a $\delta$ function in $\rho(s)$, leading to the full correlation function

$G_{2}^{\text {Full }}(t)=\frac{Z}{2 M} e^{-M t}+\xi \int_{s_{0}}^{\infty} \rho^{O P E}(s) e^{-s t} d s$.

There are four parameters in this Ansatz for $G_{2}^{\text {Full }}(t): Z$ and $M$ for the ground state, $s_{0}$ (the continuum threshold), and $\xi$. The parameter $\xi$ is introduced to allow for lattice "distortions" in the normalisation of the excited states [1]. In the continuum limit $\xi$ should be equal to one.

We have calculated the OPE expansions for all the channels studied. These are listed in [3].

\section{FITS TO DATA}

Correlation functions were fitted to the following three functional forms, $F(t)$ :

1. Conventional Single State Fit ("1-exp") i.e. using $F(t)=\frac{Z}{2 M} e^{-M t}$.

2. QCD Continuum Model Fit ("Cont") These are described in the previous section.

3. Conventional Two State Fit ("2-exp") i.e. using $F(t)=\frac{Z}{2 M} e^{-M t}+\frac{Z^{\prime}}{2 M^{\prime}} e^{-M^{\prime} t}$. This functional form was chosen since it is traditionally used as the generalisation of "1-exp" to include the higher mass state(s). It has the same number of parameters as the "Cont" fit and the results of these two fits will be directly compared.

The "1-exp" fits are used here to gain "standard values" for $Z$ and $M$ and are fitted in the asymptotic region only. In the case of the QCD Continuum Model fits, we use time windows which begin very close to the origin, e.g. $t=2-28$ for $\beta=6.0$. So that a direct comparison can be performed, the same time windows are used to perform the "2-exp" fits.

The full results of the fits to the three fitting functions for all the lattice correlation data are presented in [3]. We show in Table 1, the fits for the vector meson channel for the Wilson $\beta=6.1$ only. (The other fits follow a similar pattern.)

The main features of the fits are now summarised. The Continuum Model reproduces the data better than the 2-exp case, i.e. (i) its $\chi^{2}$ values are lower (sometimes by an order of magnitude), and, (ii) its ground state parameters $Z$ and $M$ are closer to the "1-exp" control case.

\begin{tabular}{lccc}
\hline$F(t)$ & 1-exp & Cont & 2-exp \\
\hline$Z\left(\times 10^{-2}\right)$ & $0.57(3)$ & $0.60(2)$ & $0.68(2)$ \\
$M a$ & $0.466(2)$ & $0.468(2)$ & $0.474(2)$ \\
$s_{0}[\mathrm{GeV}]$ & & $1.9(1)$ & \\
$\xi$ & & $-3.25(4)$ & \\
$\chi^{2} /$ d.o.f. & $0.4(6) / 11$ & $9(5) / 22$ & $110(20) / 22$ \\
\hline Table 1 & \\
Values for the fitting parameters for the vector \\
meson (Wilson $\beta=6.1$ case).
\end{tabular}

We also note that the 2-exp fit values overestimate $Z$ and $M$ in every channel studied. In [3] we give an explanation for this effect.

Clearly it is not sufficient for the "Cont" fits to reproduce the ground state parameters $Z$ and $M$, and to have a sensible $\chi^{2}$, they must also give values for the continuum parameters, $s_{0}$ and $\xi$ which are acceptable within the assumptions of the QCDSR Continuum Model. These are: (a) since $s_{0}$ corresponds to a physical threshold, they should be constant (in $\mathrm{GeV}$ ) for each channel as $\beta$ is varied; (b) $s_{0}$ should be large enough to be in a region where perturbation theory is valid; and (c) $\xi$ should scale like $\xi \rightarrow 1$ as $a \rightarrow 0$. It turns out that criteria (a) is satisfied for mesons, but not for baryons; that (b) is marginal; and that (c) fails totally [3]. (In fact $\xi$ can be large and negative !)

This leads us to the conclusion that the applicability of the QCDSR Continuum Model to lattice data is questionable since it leads to values for $s_{0}$ and $\xi$ which are inconsistent with the assumptions made.

It is clear that, apart from taking on nonphysical values of its fitting parameters, the "Cont" Ansatz does reproduce the lattice correlation function data. Therefore we require similar fitting Ansätze as those in the "Cont" case, but without the corresponding restrictions on the parameters $s_{0}$ and $\xi$.

\section{QUARK MODELS}

We present the derivation of the density of states, $\rho(s)$, for hadronic correlators for the nonrelativistic quark model. Taking the quarks as onshell, there are 3 degrees of freedom available for 
each quark in the hadron: one per spatial momentum component. Therefore in the case of mesons there are naively 6 degrees of freedom, with 9 degrees of freedom for baryons. However, there are 4 constraint equations corresponding to the condition that the hadron's 4-momentum is zero, leaving 2 degrees of freedom for the mesons and 5 degrees of freedom for the baryons. This simple, non-relativistic analysis leads to $\rho_{\text {nrqm }}(s) \sim s^{2}$ for mesons, and $\rho_{\text {nrqm }}(s) \sim s^{5}$ for baryons. This continuum-like behaviour for $\rho(s)$ is clearly most appropriate for large $s$ where the quarks are approximately free. Again we approximate the actual density of states by $\rho(s)=\frac{Z}{2 M} \delta(s-M)+$ $\theta\left(s-s_{0}\right) \rho_{n r q m}(s)$, where the delta-function represents the ground state and the non-relativistic quark model result is used for the continuum (beginning at the threshold energy $s_{0}$ ).

Using this definition of $\rho(s)$ in the spectral equation we obtain, for mesons,

$G_{2}(t)=\frac{Z}{2 M} e^{-M t}+K\left(\frac{1}{t^{3}}+\frac{s_{0}}{t^{2}}+\frac{s_{0}^{2}}{2 t}\right) e^{-s_{0} t}$,

where $K$ is some numerical constant, with a similar expression for baryons. It is very interesting to note that these expressions are identical to those obtained for the Cont model with $m_{q}$ and the condensate set to zero. Since the terms $\mathcal{O}\left(m_{q},\langle: \bar{q} q:\rangle\right)$ are numerically insignificant, we have shown that the non-relativistic quark model predicts essentially the same functional form as the QCDSR's Continuum Model. Thus we have achieved the aim raised in the previous Section of a (physically motivated) functional form which reproduces the data better than the "2-exp" fits, but which doesn't suffer from unphysical parameter values such as the "Cont" fit. (The relativistic quark model is discussed at length in [3].)

It is natural to wonder if there is any way of using the lattice correlation function data to pin down the best form of the density of states $\rho(s)$. With this in mind we assume that the density of states has the following form: $\rho(s)=$ $\frac{Z}{2 M} \delta(s-M)+\theta\left(s-s_{0}\right) K s^{n}$, where $n$ is to be determined from the fit. The two-point correlation function for this Ansatz is defined from the standard Laplace transform. Studying the $\chi^{2}$ values as a function of $n$ we see a clear min- imum in the case of the pseudoscalar mesons at $n=2$. The vector channel's minimum is more spread out at around $1 \leq n \leq 3$. The proton case is less clear again; it has a minimum at $n \gtrsim 5$. These results confirm the predictions of the nonrelativistic quark model.

\section{DISCUSSION \& CONCLUSION}

This work began with a study of the QCD Sum Rule Continuum Model as applied to lattice two-point correlation functions using the method first introduced by Leinweber [1,2]. We have extended his work by including mesonic states, by fitting lattice data at several lattice spacings and by using two formulations of the lattice action. We have found that the QCD Sum Rule Continuum Model successfully fits the lattice data, but only for unphysical values of its fitting parameters. This leads us to the conclusion (somewhat contrary to that of [1]) that this model cannot self-consistently fit lattice data.

We have then searched for a model that reproduces a similar functional form as the QCD Continuum Model, but which does not have its restrictions on its parameters. The non-relativistic quark model was studied and found to be such a theory. We introduced a general fitting function which has the non-relativistic quark model as a special case, and found evidence that the $\chi^{2}$ was minimized for the fitting function corresponding roughly with the non-relativistic quark model.

We propose that these fitting functions can be used to extract ground state properties in cases where the asymptotic state is heavily contaminated by excited states, or where the time separation in the correlators is forced to be small.

\section{ACKNOWLEDGEMENTS}

This work was supported by a grant form the Nuffield Foundation.

\section{REFERENCES}

1. D.B.Leinweber, Phys. Rev. D51 (1995) 6369

2. D.B.Leinweber, Phys. Rev. D51 (1995) 6383

3. C.R. Allton and S. Capitani in preparation 